\title{
Successful Remote Delivery of a Treatment for Phonologic Alexia via Telerehab
}

\author{
Getz Heidi ${ }^{a, c}$, Snider Sarah ${ }^{a}$, Brennan David ${ }^{b}$, and Friedman Rhonda ${ }^{a}$ \\ Getz Heidi: hrg2@georgetown.edu; Snider Sarah: sfs24@georgetown.edu; Brennan David: \\ david.m.brennan@medstar.net; Friedman Rhonda: friedmar@georgetown.edu \\ ${ }^{a}$ Center for Aphasia Research and Rehabilitation, Georgetown University Medical Center, 4000 \\ Reservoir Road NW, Building D 207, Washington, DC 20007 \\ bMedStar Institute for Innovation, 3007 Tilden Street, NW, Washington DC 20008 \\ 'Heidi Getz is now at the Center for Brain Plasticity and Recovery, Georgetown University Medical \\ Center
}

\section{Abstract}

A growing body of literature supports the effectiveness of the remote delivery of rehabilitation services, i.e., telerehab. Aphasia treatment is particularly well suited for telerehab because of the verbal and visual nature of speech-language therapy, but scientific research investigating aphasia telerehab is in its infancy. No studies to date have evaluated whether treatment of acquired reading disorders by a live clinician can be feasibly, effectively, or efficiently conducted via telerehab. Here we address this gap in the literature by reporting our success remotely remediating the reading deficits of two participants with phonologic alexia. We adapted for the telerehab setting a previously validated treatment for phonologic alexia (Friedman, Sample, \& Lott, 2002), which uses a paired-associate design to train reading of problematic words. Both telerehab participants significantly improved their reading of trained words in similar time frames as previous participants (Friedman et al., 2002; Kurland et al., 2008; Lott, Sample, Oliver, Lacey, \& Friedman, 2008); furthermore, both participants reported high satisfaction with the telerehab setting. Although telerehab with alexic patients poses unique challenges, we conclude that treatment for alexia via telerehab is nevertheless feasible, may be equally effective as in-person treatment, and saves substantial resources for participants as well as clinicians.

\section{Keywords}

aphasia; alexia; treatment; telerehabilitation; videoconferencing

\section{Introduction}

The demand for speech and language rehabilitation services is growing as our society sees a larger number of patients aging with a disability (i.e. as a result of improved treatment for stroke and brain injury). Because of this increasing demand, as well as clinician shortages, 
decreased reimbursement, and shorter lengths of stay at outpatient rehabilitation facilities, rehabilitation practitioners are turning to telemedicine in an effort to improve access, enhance the quality of care, and reduce costs (Brennan \& Barker, 2008). Through modern information and communication technologies, services can be delivered to patients who would otherwise be unable to receive them due to distance from a healthcare facility, lack of trained clinicians in a geographic area, logistical obstacles to travel, or mobility impairments.

Aphasia treatment in particular is ideally suited for telerehab because of the verbal and visual nature of language and reading therapies (Brennan, Georgeadis, \& Baron, 2002). Models of aphasia telerehab can be grouped into three main categories according to the nature of interaction between the clinician and the person with aphasia (PWA): synchronous, in which treatment is conducted "live" through a real-time audio/video connection; asynchronous, in which the PWA completes treatment exercises independently and the clinician monitors results and provides feedback 'offline'; or independent, in which the PWA participates in a form of computer-based therapy independently, without clinician oversight.

Synchronous aphasia telerehab provides the closest parallel to traditional rehabilitation and has been successful, in terms of clinical efficacy as well as patient satisfaction, with a variety of patient populations and clinical deficits. It was first implemented via telephone to treat acquired communication deficits in veterans (Vaughn, 1976). Technological advances have facilitated the expansion of synchronous telerehab to treatment of dysarthria in Parkinson's disease (Tindall, Huebner, Stemple, \& Kleinert, 2008), stuttering in children and adults (Kully, 2000), and apraxia in stroke survivors (Cavin-Root, Lasker, Spence, \& Stierwalt, 2010). With PWA, telerehab has focused on diagnosis and assessment of language profiles; these studies have demonstrated clinical equivalence of in-person and synchronous telerehab assessment, even for moderate to severe aphasia (Georgeadis, Brennan, Barker, \& Baron, 2004; Hill, Theodoros, Russell, \& Ward, 2009; Theodoros, Hill, Russell, Ward, \& Wootton, 2008).

Relatively few investigations of synchronous aphasia telerehab have explored actual treatment. Those that have done so have largely focused on remediating lexical retrieval deficits (Agostini et al., 2014; Dechêne et al., 2011; Furnas \& Edmonds, 2014). For example, Dechêne and colleagues (2011) treated anomia in three post-stroke PWA using a synchronous audio/video connection and tablet PC. Pairs of target items were chosen on the basis of participants' baseline performance; from each pair, the participant selected the item s/he wished to train on. Following six weeks of training on a variety of reading, typing, and naming tasks, participants demonstrated a "clinically relevant" improvement for trained items and less for untrained items. Although methodological weaknesses in this study (particularly the selection of treatment words) preclude strong conclusions about the efficacy of its treatment paradigm, all three participants expressed high satisfaction with telerehab. More recently, Agostini and colleagues (2014) reported a well-controlled study directly comparing treatment outcomes for synchronous aphasia treatment in-person and via telerehab. Lexical retrieval deficits in five post-stroke PWA were treated using a phonemic cueing paradigm. Participants trained on one word list in-person and a second list via Skype, with the order of treatment settings counterbalanced across subjects. Significant 
improvement in naming accuracy of trained items was observed for all participants, did not decline significantly at follow-up testing three weeks later, and crucially, was equivalent across treatment settings.

A second form of aphasia telerehab uses 'store-and-forward' technology (Baron, Hatfield, \& Georgeadis, 2005), which incorporates asynchronous clinician-patient interaction. In asynchronous telerehab, participants' responses on computerized tasks are stored and made accessible to clinicians who may then remotely monitor progress, modify therapy materials, or adjust task difficulty. An early investigation by our own group incorporated a store-andforward component into a successful treatment for pure alexia (Friedman \& Lott, 2000). The store-and-forward model has also been used successfully in the treatment of anomia, in particular by Mortley and colleagues (Mortley, Wade, Enderby, \& Hughes, 2004; Mortley, Wade, \& Enderby, 2004). Those researchers successfully implemented remote anomia therapy for seven post-stroke PWA using the software program StepByStep. Participants' responses on treatment exercises (spoken production, typed spelling, or multiple-choice answers) were forwarded electronically to the clinician, who then followed up with the participant via phone for discussion. Adjustments to exercises were uploaded by the clinician remotely, then downloaded to the computer by the participant. Naming performance improved post-treatment, supporting asynchronous aphasia telerehab as effective and feasible.

A third form of aphasia telerehab involves treatment software - either web-based or downloadable - that PWA complete independently, in the absence of a clinician. One such program, ReadRight, has been demonstrated to improve reading speed in alexia (Ong et al., 2012). Improvement in language abilities has also been demonstrated with the web-based treatment Oral Reading for Language in Aphasia (ORLA), a synchronous/independent hybrid form of telerehab (Cherney, Kaye, \& Hitch, 2011; Cherney, Babbitt, et al., 2011). In this study, 32 PWA were randomized to receive either a previously validated web-based treatment for aphasia or (in the control condition) to play a non-verbal, commercially available computer game. In treatment, participants read sentences along with a "virtual therapist" avatar which provided visual speech modeling and verbal encouragement. The clinician occasionally monitored treatment sessions through audio/video feeds of each participant, and could interact directly with PWA via chat messaging. At post-testing, both groups' language had significantly improved, which was interpreted as evidence supporting the potential of nonverbal, attention-demanding computer games in the remediation of language deficits.

Asynchronous and independent models of aphasia telerehab shift control over treatment including schedule, session duration, and even choice of treatment exercise - to the PWA (Mortley, Wade, \& Enderby, 2004). Asynchronous (store-and-forward) models also translate to substantial time savings for clinicians, whose time conducting live therapy is drastically reduced, thereby freeing up time for therapy planning and treatment of additional patients. These features allow for a less resource-intensive means of delivering high-volume, highfrequency interventions. However, asynchronous and independent models of telerehab by definition lack live interaction with trained clinicians, and for this reason may be most powerful as an adjunct to clinician-directed care rather than as a replacement. 
Synchronous telerehab, on the other hand, combines the advantages of the clinician's expertise (including real-time live interaction and feedback), the convenience of at-home sessions, and the data tracking capabilities of computer-based treatment. In this sense, synchronous telerehab may in some cases be an improvement on in-person therapy: by removing the need for patients to physically travel to receive treatment, clinicians can more easily adjust treatment frequency or deliver follow- up 'booster' sessions, and patients can receive treatment while on vacation, in the workplace, or when too ill to come in for a session. Indeed, Goldberg and colleagues have successfully used telerehab to increase treatment session frequency in a script training intervention (Goldberg, Haley, \& Jacks, 2012). Two PWA were trained on personally relevant dialogue scripts, primarily via synchronous telerehab with some in-person sessions. Treatment incorporated a cuing hierarchy paradigm, written scripts to be read by participants, and audio recordings for independent use by the participant in practice sessions. Probe testing without cues but with the written script was conducted at the start of each telerehab session. Results of this multiple-baseline study revealed improvement in multiple domains of speech, including accuracy, rate of speech, and articulatory fluency. Importantly, no difference was observed in probe performance in-person as compared with telerehab.

The studies reviewed here comprise a small but growing body of scientific evidence supporting the efficacy and feasibility of synchronous aphasia telerehab. The scope of these findings, however, remains quite narrow. Although a small number of studies have targeted the treatment of language deficits in general (Cherney, Kaye, et al., 2011; Goldberg et al., 2012), the majority have focused on the common but rather circumscribed deficit of anomia (Agostini et al., 2014; Dechêne et al., 2011; Furnas \& Edmonds, 2014). Our study aimed to address a critical need in the literature for evaluating the effectiveness and efficacy of a broader range of treatment protocols delivered via telerehab. In particular, although remote alexia therapy using store-and-forward technology has previously been successfully implemented - including by our own group (Friedman \& Lott, 2000) - interactive, synchronous sessions treating alexia via telerehab has to our knowledge never before been attempted.

This gap in the literature is no coincidence: the challenges of this patient population make remote treatment of any deficit particularly intimidating. Even validated telerehab interventions for non-reading deficits such as anomia may not be accessible to patients whose anomia is accompanied by alexia. For example, therapies that involve reading sentences and paragraphs (Cherney, Kaye, et al., 2011; Goldberg et al., 2012) are ill-suited to PWA who struggle to read even single words. Similarly, tasks involving typed responses (Dechêne et al., 2011; Furnas \& Edmonds, 2014) are not appropriate for PWA with impaired letter identification abilities (unless those PWA happen to be touch-typists). Even when therapies are accessible, certain aspects of computerized treatment may pose particular challenges for alexic participants, including navigating the menus of an unfamiliar computer; handling unexpected error messages; entering a simple password to dictation; and so on. These challenges occur on top of those associated with their aphasic status more generally, e.g. the auditory comprehension and word-finding deficits that render phone communication difficult. It is therefore an open question whether synchronous telerehab of 
severely alexic patients is even feasible, let alone effective. The research reported here was designed to answer this question.

\section{Methods}

\section{Treatment Intervention}

The intervention reported here aims to treat phonologic alexia, a reading disorder that may result from left hemisphere stroke. This disorder is characterized by marked difficulties reading words with little to no semantic content, such as function words, some verbs, and especially novel words or pseudowords, as compared with relatively preserved reading ability for concrete nouns and adjectives. One successful treatment for phonologic alexia, designed by our own group (Friedman et al., 2002), pairs words that the patient has difficulty reading (e.g. would) with homophonic or near-homophonic words with greater semantic content (e.g. wood). The goal of this paired-associate paradigm is to circumvent the impaired orthography-to-phonology reading route by training reading to proceed via the preserved semantic knowledge system. We have repeatedly obtained successful results with this paradigm in the past (Friedman et al., 2002; Kurland et al., 2007; Lott et al., 2008). In brief, participants are trained on a set of problematic target words and a set of phonologically related words that serve as paired associates. Word pairs are trained via paper flashcards, the front of which lists the target word and the back of which lists the target word, its paired associate word, and the associate's picture. Oral reading of the target is achieved by reading, naming, or repeating the phonologically related paired associate followed by the target. Improvement is measured as ability to read the target word in the absence of the semantic cue. In the current study, we replaced paper flashcards with "virtual flashcards" presented remotely via custom software and a videoconferencing system.

\section{Participants}

Participants who met our criteria for phonologic alexia (see below) were recruited from a local stroke support center. Georgetown University's Institutional Review Board approved this study, and signed informed consent was obtained from each participant prior to baseline testing.

Participant 1-BLN is an African-American man, age 44 at the time of this study, who completed two years of college. Prior to his stroke, he worked as a real estate agent. Fifty-six months prior to participation in this study, BLN suffered a large hemorrhage in the area of the left middle cerebral artery, resulting in a lesion involving portions of the left frontal, temporal, and parietal lobes.

Participant 2-ESM is an African-American woman, age 51 at the time of this study, with a high school-level education (GED). Prior to her stroke, she worked as a receptionist for a law firm. Baseline testing for this study began approximately 44 months after her stroke. An MRI scan soon after her stroke revealed a large infarction of the left middle cerebral artery involving a small portion of the left temporal lobe and a large part of the left frontal and parietal lobes. 
Neuropsychological evaluation-General cognitive abilities were assessed using the Ravens Colored Progressive Matrices (RCPM) and the Biber Figure Learning Test (BFLT). On the RCPM, BLN's score of $34 / 36$ was above the $95^{\text {th }}$ percentile, while ESM's score of $31 / 36$ fell between the $50^{\text {th }}$ and $75^{\text {th }}$ percentile for adults of her age. On the BFLT, scores for both BLN and ESM were within the range of normal. Both participants scored $100 \%$ on a line cancellation task designed to test for neglect. Spatial span for both participants was at the $50^{\text {th }}$ percentile (BLN: forward 5, backward 6; ESM: forward 5, backward 5) while digit span was severely impaired (BLN: forward 4, backward 3, $2^{\text {nd }}$ percentile; ESM: forward 3, backward $0,<1^{\text {st }}$ percentile).

Language evaluation-At the time of this study, BLN presented with a chronic severe nonfluent aphasia as assessed by a trained speech-language pathologist. Language profile as measured by the Boston Diagnostic Aphasia Examination (BDAE) was characterized by severely decreased fluency with a typical phrase length of 2 words; impaired nonverbal, verbal, and articulatory agility; moderately impaired auditory comprehension; and poor reading abilities. Repetition was good for one-syllable words, but broke down with additional syllables. BLN could not repeat any sentences. Confrontation naming was poor (Boston Naming Test (BNT): 21/60) with frequent phonologic and occasional verbal paraphasias.

ESM also presented with a chronic severe nonfluent aphasia. As assessed by the BDAE, ESM's language profile was characterized by decreased fluency, with a typical phrase length consisting of 2 words, and impaired verbal (but intact nonverbal) agility. She correctly repeated 7/10 single words but was unable to repeat any sentences. Confrontation naming was severely impaired (BNT 10/46, testing discontinued); error responses included both phonological and verbal paraphasias. ESM's auditory comprehension was good for functional, contextual speech but impaired for lengthy and more complex information.

Letter knowledge: Letter naming was impaired for both participants (lowercase letters named correctly: BLN 18/26, ESM 5/26). Letter identification (pointing to a named letter in an array of 6) was also impaired, with BLN correctly pointing to 16/26 lowercase and 22/26 uppercase letters and ESM correctly identifying 17/26 lowercase and 17/26 uppercase letters. Participants performed well on a two-alternative forced-choice task requiring them to determine whether a letter was in its correct or mirror-image orientation (51/52 for both participants). Finally, both participants scored $100 \%$ on a task requiring a same/different judgment for pairs of letters in different cases (one lowercase, one uppercase).

Oral Reading Analysis: Oral reading ability was assessed with a set of words of differing parts of speech, matched on several relevant variables, including frequency and word length. ${ }^{1}$ Both participants demonstrated a substantial and statistically significant part-of-speech effect in oral reading, with significantly better performance on concrete nouns and adjectives as compared with abstract nouns, verbs, and functors on a 205-item list (Figure 1; concrete versus abstract nouns, BLN $\left.\chi^{2}=3.87, \mathrm{p}<.05 ; \operatorname{ESM} \chi^{2}=9.77, \mathrm{p}<.01\right)$. Neither participant

${ }^{1}$ Matching frequency across word categories is not possible on a list of words of different parts of speech. However, such frequency confounds produce an advantage for functors, i.e., exactly those words that are most difficult for phonologic alexics. 
was able to read three- or four-letter pronounceable pseudowords (PWs) (BLN: 1/20, ESM: 0/20). Both participants had significantly more success reading a list of real words (RWs), each of which differed from an item on our PW list by only a single letter (BLN: 14/20, ESM: 10/20) (PWs vs. RWs: BLN $\chi^{2}=18.03$; ESM $\chi^{2}=13.33$, both $\left.\mathrm{p}<.001\right)$. On the basis of these results, both BLN and ESM were judged to meet our criteria for phonological alexia.

Phonology: Both participants demonstrated difficulty on a PW repetition task. BLN correctly repeated 5/10 one-syllable, 3/10 two-syllable, and 0/10 three-syllable PWs. ESM correctly repeated only $3 / 10$ one-syllable words. ${ }^{2}$

Semantics: A test of access to conceptual knowledge from pictures of objects (Pyramids \& Palm Trees, Howard \& Patterson, 1992) revealed no impairment for BLN (score: 49/52) and mild impairment for ESM (score: 47/52).

Spelling: Both participants performed very poorly on a spelling test of 3- and 4-letter PWs (BLN: 0/10, ESM: 1/20). Their performance improved but remained poor with 3- and 4letter RW's (BLN: 9/20, ESM: 11/20). Both participants were better able to spell nouns and adjectives than verbs and functors (BLN: 9/16 nouns and adjectives, 0/16 verbs and functors; ESM: $15 / 16$ nouns and adjectives, 7/16 verbs and functors).

\section{Study Design}

Initial language and neuropsychological evaluation as well as baseline testing of each participant was conducted in-person at the Center for Aphasia Research and Rehabilitation (CARR) at Georgetown University Medical Center over several test sessions, during which time the treatment stimuli were chosen (see Stimuli). Participants subsequently returned to CARR for a one-session demonstration of the telerehab equipment (see Telerehab System). On a separate day, researchers brought the telerehab equipment to the participant's home, set it up, and initiated a test connection to other researchers at CARR. All treatment sessions were conducted remotely (telerehab). Post-treatment assessment was conducted in-person at CARR.

\section{Telerehab System}

Following language and neuropsychological evaluation and baseline testing, participants were invited individually to CARR for an individual demonstration and instruction on use of the software. One researcher used the "clinician" computer to connect to the participant, who was located (along with a second researcher) several offices away so as to simulate the home setting. The participant was shown how to power on the participant laptop and enter the software system as described below. The researcher at the "clinician" computer then established a connection to the participant and briefly demonstrated the program. At a later date, researchers set up the telerehab equipment at participants' homes, connected to their home wireless networks, and assisted in establishing a connection with the researcher at CARR. The CARR researcher then conducted the first full treatment session, while a

\footnotetext{
${ }^{2}$ Because of her poor performance on 1-syllable PW's, ESM was not asked to attempt any longer PWs.
} 
researcher remained at the participant's home to troubleshoot and address any potential technological problems or provide additional user training.

Equipment and software-Each participant was provided with an IBM ThinkPad equipped with a hands-free noise cancellation microphone and a built-in video camera, to be used exclusively for the research investigation. Participant computers were pre-loaded with the custom "home practice" software developed for the treatment protocol, Vidyo videoconferencing software (vidyo.com), and the application LogMeIn (logmein.com), which allowed researchers to control participants' computers remotely. The "clinician" system consisted of a Dell 790 OptiPlex desktop computer loaded with the treatment software and Vidyo; an add-on video camera; and a hands-free noise cancellation microphone.

Treatment sessions proceeded as follows: Participants powered on the computer and clicked the "Participant" user icon to $\log$ in. A password was not required. To connect to the researcher, the participant clicked on a desktop shortcut to enter a designated, private virtual "room" in Vidyo, typed their name or initials, and clicked "Join". A video feed of the researcher (if she had already connected) or the participant (if the researcher had not yet connected) then appeared on the screen. The researcher accessed the Vidyo "room" in the same manner. Once both the researcher and the participant had connected through Vidyo, they could view one another's video feeds in real time and engage in conversation. To begin the session, the researcher opened the custom treatment application (located on the "clinician" computer) and used Vidyo's screen-sharing feature to allow the participant to view the treatment software. In this manner, the researcher navigated through the software while controlling the display on the participant's screen. Throughout this process, picturebased instructions with minimal text were available to the aphasic participants as well as the researchers.

Internet connection-Both participants had wireless Internet access in their homes prior to enrollment in this study. We asked them to conduct a network test to determine the speed of their broadband connection using an online bandwidth utility (http://www.att.com/ speedtest/). Both connections (BLN: Comcast 11.36 Mbps download, 4.03 Mbps upload; ESM: Verizon 29.46 Mbps, 24.32 Mbps) were considered to be sufficient to allow for a high-quality video and audio feed that would facilitate an appropriate level of interaction during the telerehab sessions. The researchers relied on Georgetown University Medical Center's network connection.

\section{The Treatment}

Stimuli-Two hundred and fourteen functors and verbs that could be paired with a pictureable noun homophone were selected as candidate treatment items from the materials used in previous studies (Friedman et al., 2002; Lott et al., 2008). As part of the initial evaluation conducted in-person at CARR, all 214 words were presented to each participant for oral reading on three separate occasions, in different pseudo-randomized orders, at least 1 week apart. The words that each patient was unable to correctly read aloud on at least 2 of 3 baselines (BLN: $n=82$, ESM: $n=171$ ) were identified as candidate treatment items. These 
items' corresponding homophone or near-homophone relay words were then presented to participants during a separate baseline testing session for oral reading. If the participant failed to read a relay word correctly, the researcher read it aloud and asked the participant to repeat it. Accuracy, including whether repetition of the word was required for correct production, was recorded.

Of the candidate treatment items whose relay words were correctly read or repeated, three sets of 20 words were selected (two sets to be trained and one to remain untrained). The word sets were initially balanced for average number of syllables and letters using the Match program (Van Casteren \& Davis, 2007). We then manually edited word sets to ensure that the final lists were matched as closely as possible for overall reading accuracy at baseline. The status of relay words as exact (e.g. be/bee) or near (bind/binder) homophones was balanced for all three sets for BLN and for the two to-be-trained sets (i.e., those for which the relay word would be used) for ESM. In addition, the number of relays requiring repetition for accurate production (BLN only) was balanced across sets. ESM's sets were also matched for number of pronouns, number of past-tense verbs, and number of words beginning with two consonants. Selected set parameters are presented in Table 1, and the full list of target items appears in the appendix. Sets 1 and 2 were trained sequentially for each participant; set 3 was not trained. This design ensures that improvement on Sets 1 and 2 can be attributed to the initiation of treatment, rather than to spontaneous improvement over time.

Procedure-Telerehab was conducted three times per week for approximately 45-60 minutes. Participants were also instructed to complete home practice twice per week. Each telerehab session began with an oral reading probe of the set of words currently in treatment. Target words were presented individually on the shared screen in one of eight pre-set, pseudorandom orders, and participants were asked to read aloud each word. The set of treatment words not currently being trained was probed once per week (i.e. every third session). On days when both treatment sets were to be probed, sets were probed in alternating order.

Evaluation was conducted primarily by the speech-language pathologist, and telerehab was conducted by the speech-language pathologist (S.F.S) or a second researcher (H.R.G.). When possible, both researchers were present for probe testing, and each researcher independently recorded oral reading accuracy while observing the participant via video feed. This second-rating of response accuracy was conducted because the participants' articulatory deficits rendered it difficult, at times, to clearly perceive their responses through telerehab, despite good-quality video and audio signal. A response was considered to be incorrect if either experimenter noted it as incorrect, thus applying the strictest possible criteria for a correct response.

Following probe testing, each session advanced to treatment. Targets were presented individually for oral reading. If the response was incorrect or distorted, the experimenter revealed the homophone or near-homophone relay word and corresponding image by clicking the "Flip" button in the telerehab software (Figure 2), which simulates the process of turning over the paper flashcard. The participant was then instructed to read the relay 
word, followed by the target word. If the participant was unable to read the relay word, the researcher read it aloud and asked the participant to repeat it. During the first treatment session for each set, relay words were revealed and read/repeated by the participant regardless of target item accuracy. The following information was recorded about each treatment trial: whether the target was read correctly, whether presentation of the relay word was required, and whether the relay word (if revealed) was read correctly. The total number of runs through the treatment sets in a given session was also recorded. Both participants averaged approximately four runs per telerehab session.

At the conclusion of a telerehab session, the researcher selected a set for home practice (Figure 3) and clicked "OK" to remotely program the participant's home-practice software to display that set when next opened. The procedure for home practice was the same as the telerehab treatment, except that the participant clicked the "flip" button him/herself, as needed or desired. The software was programmed to complete three randomized cycles of the programmed set, generate an email to the research staff containing start and end time of the practice session and the list of cards that were "flipped," and exit automatically. More detailed log files containing date and time information for each action taken while using the software were saved locally on participants' computers and retrieved at the conclusion of the study.

Participants progressed to Set 2 after reaching 90\% accuracy on Set 1 in two consecutive probe tests or after performance on Set 1 reached a plateau. A plateau was defined as a change of five percent (i.e. one item of the 20 -item list) or less over six sessions. Once the participants reached $90 \%$ accuracy on two consecutive probes of set 2 , or performance reached a plateau, the telerehab portion of the study concluded and participants returned to the lab for in-person post-testing. Satisfaction with the telerehab system was assessed by the speech-language pathologist in an individual interview, guided by a structured questionnaire designed to assess the participant's experience with the software from both a clinical and a technological perspective.

\section{Results}

\section{Inter-Rater Reliability}

Eighty-five percent of the probe tests were second-rated. Inter-rater reliability was good, with raters agreeing on an accuracy score $96 \%$ of the time for ESM and $94 \%$ of the time for BLN.

\section{Treatment Intervention}

Behavioral outcome-ESM required nine sessions (five telerehab and four home practice) to reach criterion on Set 1, and six sessions (four telerehab and two home practice) to reach criterion on Set 2. BLN did not reach criterion on either set, although he did significantly improve his reading of the trained words as assessed post-treatment (see below). He reached a plateau of 74\% correct on Set 1 after 14 sessions (nine telerehab and five home practice) and 78\% correct on Set 2 after nine sessions (seven telerehab and two home practice). 
Oral reading accuracy of target words post-treatment was assessed in the same way as at baseline: in the context of the larger list of 214 candidate items, on three separate occasions throughout the post-treatment assessment period (within a time span of 10 days for ESM and 22 days for BLN). Accuracy results for trained items on each of the three pre-treatment baseline tests, on all probe tests conducted throughout treatment, and on each of the three post-treatment baseline tests are presented in Figure 4.

Oral reading accuracy pre- and post-treatment for trained as compared with untrained items is displayed in Figure 5 as summed accuracy across all three presentations of the 214-item list. Both participants demonstrated significant improvement on the two trained sets (Wilcoxon signed-rank test, all p's < .001) and minimal improvement on the third, untrained set. We assessed reading accuracy across three time points pre-treatment to ensure that the words selected for training were reliably difficult for individual participants, rather than only sporadically incorrect. We assessed accuracy in the same way post-treatment in order to maximize comparability with our pre-treatment measures. Performance on all three sets remained stable across all three post-treatment tests for both participants (Friedman Test, all p's > .1).

Comparison with in-person treatment-The number of sessions required by BLN and ESM to reach criterion or plateau on the treatment sets was compared to the number of sessions required by previous participants to complete the semantic mediation treatment inperson (Friedman et al, 2002; Lott et al, 2008; Kurland et al, 2008). As depicted in Figure 6, both BLN and ESM performed similarly to previously reported participants. In fact, ESM learned to read her Set 1 words even faster than any of the three participants who received treatment in-person (YCR, DN, and $\mathrm{HN}$ ).

\section{Telerehab System}

Participant satisfaction-Both participants expressed high satisfaction with the telerehab system in the post-telerehab interview. BLN and ESM were asked to rate the telerehab sessions as better, the same, or worse than the in-person assessment sessions on the following factors: the researcher's understanding of the PWA, the PWA's understanding of the researcher, the PWA's level of concentration, the PWA's environment, and the quality of communication. Both participants indicated that telerehab was "the same" as in-person sessions in each of these areas. Participants were also asked which method of delivery they would choose (in-person or telerehab) if they could receive the same treatment again. BLN responded that he would choose the computerized version. ESM said "I like you [the SLP], but the computer is fine too." When asked what they liked about the telerehab technology, ESM described the software as "excellent", and BLN indicated that he liked that he did not have to travel. Neither participant reported disliking anything in response to direct questioning.

Performance of the telerehab system-The researchers found the telerehab system user-friendly, easily navigable, and most important, well equipped to handle unexpected technological problems. For example, at one point, ESM communicated to a researcher that her computer was "dark", by which the researcher inferred that the screen had auto-dimmed 
due to lack of mouse or keyboard activity. On another occasion, it was discovered that Vidyo's echo cancellation setting had somehow turned off, leading to distracting voice feedback. These minor problems were easily resolvable using LogMeIn; in each case, following adjustment of the settings, the treatment session proceeded as usual. The most substantial obstacle occurred when a researcher encountered an error message indicating that user interaction was required for a mandatory software update to Vidyo. Installing this update required clicking a button, waiting for the file to download, executing the file, and relaunching the application. Although trivial for the researcher to accomplish, attempts to guide participants through this process over the phone proved far more challenging. Additionally, although we had been careful to avoid requiring a password to log into the computer, security settings had intentionally been selected to require a password for any changes to the system. (This was a security measure in place to protect our equipment from modification by participants and their family members.) Although the researcher successfully guided participants over the phone to download the update, both participants' attempts to type the administrator password to dictation were unsuccessful as a result of their impaired letter identification abilities. The application LogMeIn proved to be crucial in resolving this issue: it enabled the researchers to log into the participant computers, type the administrator password, and execute the updates.

\section{Discussion}

This paper has reported our success remotely delivering SemMed, a validated treatment for phonologic alexia. Two participants improved their reading of forty problematic target words in a treatment intervention conducted remotely using videoconferencing, custom software, and the remote-control application LogMeIn. We suggest that this improvement reflects successful mapping of target items' orthographic representations to their phonological representations, through association of target items' written word forms with those of their phonologically related but semantically well-represented paired associates. Establishing this association does not require orthography-to-phonology mapping (which is fundamentally deficient in phonologic alexia), nor does it rely on intact repetition abilities (lacking in our participants); its main requirement is the intact ability to access the phonologic representations of semantically rich words. Our results show that reading in phonologic alexia can be remediated via this mechanism just as well via synchronous telerehab as it is in-person, providing the first demonstration to date that live, synchronous treatment of acquired reading deficits by a trained clinician can be feasibly, effectively, and efficiently conducted remotely.

\section{Behavioral outcomes}

Both participants' oral reading of trained items improved significantly following treatment, while minimal improvement was observed on a matched set of untrained words. This result mirrors outcomes from prior studies of in-person SemMed (Friedman et al., 2002; Kurland et al., 2008; Lott et al., 2008). Although we did obtain positive treatment effects for both participants, BLN and ESM differed somewhat in pace and magnitude of improvement. While BLN's reading of target items improved substantially and significantly, his performance plateaued at $75-80 \%$ accuracy on both sets. In contrast, ESM reached (in fact, 
surpassed) our criterion of $90 \%$ correct on both sets of target words, and did so in fewer sessions than it took BLN to reach plateau. There are several possible explanations for this difference in performance. Participants' language and reading profiles may have played a role. For example, because SemMed was designed for patients with strong effects of word concreteness on oral reading performance (i.e., patients with phonologic alexia), the best results might be expected for PWA with more pronounced effects of word concreteness (like ESM). Degree of improvement as measured here may also be influenced by articulatory abilities, since patients with somewhat more impaired articulation (like BLN) may struggle to consistently produce words clearly enough to reach criterion. ESM's greater improvement could also stem from the superiority of her relay words (nearly 80\% of ESM's relay words were exact homophones, as compared with approximately $50 \%$ for BLN).

Importantly, the computerized setup of our treatment intervention allows us to investigate an additional explanation for our participants' difference in performance. Unlike with the traditional approach to this treatment (providing participants with paper flashcards for home practice), the computer-assisted method used here enabled close tracking of home practice behavior, including information regarding whether, when, and for how long home practice was in fact completed. This information may arguably serve as an indirect measure of participant motivation, effort, and/or engagement with treatment. ESM completed home practice two times per week, as directed. BLN completed home practice a total of seven times over 5.5 weeks, skipping four sessions. In addition to practicing more frequently, ESM's sessions were also slightly longer: she averaged 13.7 minutes per home-practice session, whereas BLN averaged 11 minutes per session, although this difference was not statistically significant $(\mathrm{p}>.1)$. It is therefore possible that ESM's more frequent completion of home practice - and/or the effort and motivation underlying her home-practice behavior - resulted in better learning of the trained items. We are able to entertain this interesting explanation as a result of the detailed tracking of usage statistics enabled by the telerehab software, underscoring the utility of this feature in clinical practice.

\section{Telerehab system}

Both aphasic participants expressed satisfaction with the telerehab system and were clearly comfortable using it. Some challenges did result from their alexia (notably, dealing with error messages and typing passwords to dictation); however, these situations were easily and quickly resolved through use of the remote-control software LogMeIn. We believe that our success is due in large part to the explicitly and purposefully aphasic- and alexic- friendly design of the system: we emphasized icons rather than text; treatment software was controlled by the researcher, not the participant, minimizing any need for the participant to interact with the computer; and we installed the remote-control application as a safeguard against unanticipated problems.

Most importantly, the telerehab setup of our study enabled these two PWA to participate in a relatively time-intensive treatment protocol when they might otherwise not have been able to. Table 2 details the time and distance required of each participant to travel to CARR, as well as the substantial savings enabled by telerehab. In addition to the raw time commitment of the trip to and from CARR, participants faced logistical obstacles to travel, including 
weekly obligations and family commitments. Although BLN was able to drive himself to inperson sessions, ESM was not, and relied for transportation instead on the availability of her son, who worked full-time. In-person sessions obviously required a significant investment of time and energy for both participants; without telerehab, consistent attendance at thriceweekly treatment sessions may not have been possible. Telerehab, however, allowed them to incorporate treatment into their daily lives with minimal interruption to their routine.

\section{Future Directions}

The telerehab system used in our study was relatively simple in both therapy and software design: it involved simple visual presentation of stimuli, no kinesthetic components, and no special timing needs. However, aphasia and alexia rehabilitation protocols are frequently far more intricate, and some researchers have concluded that some "hands-on" rehabilitation protocols, such as those involving tactile feedback, may not be possible via telerehab (e.g. Mashima \& Doarn, 2008, p. 1105). At the same time, treatments for aphasia, alexia, and anomia that make use of kinesthetic tactics such as tracing letters or writing words are widespread, and have been shown to be quite effective (Lott, Carney, Glezer, \& Friedman, 2010). It is therefore imperative to consider how telerehab might be adopted to incorporate these evidence-based treatments. For example, telerehab systems need a mechanism for rapid electronic transmission of traced, drawn, and written responses of PWA. We are currently investigating the use of electronic signature pads for this purpose in a remotely implemented, orthography-based treatment for anomia in primary progressive aphasia (PPA), a neurodegenerative condition. These signature pads transmit the participant's written response to the experimenter's computer screen in real time and allow the experimenter to save the completed written response as an electronic image file. Other common aphasia rehabilitation techniques that will require investigators' and clinicians' creativity for remote implementation include paradigms with precise timing constraints or computer-based auditory feedback.

A second area for future research concerns the feasibility of telerehab when aphasia is more severe. In the current study, our participants' familiarity with technology and relatively intact cognitive abilities presumably contributed to the ease with which they navigated the telerehab software. Further research will be necessary to determine whether individuals less familiar with technology, or suffering from more generalized and/or severe cognitive deficits, will be equally at ease with the software. Our PPA telerehab study (described in the preceding paragraph) is aimed in part at addressing this question. In the early stages of PPA, deficits are by definition limited to the domain of language, but impairment in other cognitive domains surfaces as the disease progresses. As we conduct speech, language, and neuropsychological assessment throughout this study's 30-month duration, we will determine whether accurate evaluation, in addition to effective treatment, is affected by multi-domain cognitive decline in this challenging population. We will also learn whether later-stage PPA patients will continue to be able to navigate the telerehab system, independently or with assistance. This ongoing investigation will thus help reveal the capabilities and, perhaps, limitations of telerehab with aphasic and neurodegenerating populations. 


\section{Conclusion}

Recent years have brought great progress in the field of aphasia telerehab, as researchers have begun to move beyond assessment and into the use of telerehab to effectively remediate, from a distance, the language deficits of PWA. However, significant work remains. The body of research describing effective in-person therapies for aphasia, alexia, and anomia is large, and treatment approaches are diverse and numerous. Although many such treatments, such as those that are solely verbal, are easily implemented remotely, other treatments are not as telerehab-friendly. Yet these more complicated protocols may be the most effective treatment for certain language and reading deficits. The task before us now is to determine how these more complex therapies could be implemented remotely, and to successfully do so, thus validating these approaches for use via telerehab by clinical SLP's. Progress of this type will require interdisciplinary collaboration among speech-language pathologists, researchers, software engineers, and PWA themselves, and will benefit from innovative re-purposing of ongoing technological advances. These contributions to the scientific literature on telerehabilitation in aphasia treatment hold the potential to vastly expand access to badly needed language rehabilitation services.

\section{Acknowledgments}

This research was supported by a Clinical and Translational Research Award to R. Friedman from NCATS grant UL1RR031975. The authors would like to thank Ming-Jye Hu for her assistance with study implementation, BLN and ESM for their dedication to our project, and two anonymous reviewers for their helpful comments.

\section{References}

Agostini M, Garzon M, Benavides-Varela S, De Pellegrin S, Bencini G, Rossi G, Tonin P. Telerehabilitation in poststroke anomia. BioMed Research International. 2014; 2014:706909.10.1155/2014/706909 [PubMed: 24829914]

Baron C, Hatfield B, Georgeadis A. Management of communication disorders using family member input, group treatment, and telerehabilitation. Topics in Stroke Rehabilitation. 2005:49-56. [PubMed: 15940584]

Brennan DM, Barker LM. Human factors in the development and implementation of telerehabilitation systems. Journal of Telemedicine and Telecare. 2008; 14:55-58. [PubMed: 18348747]

Brennan DM, Georgeadis A, Baron CR. Telerehabilitation tools for the provision of remote speechlanguage treatment. Topics in Stroke Rehabilitation. 2002; 8(4):71-78. [PubMed: 14523731]

Cavin-Root C, Lasker JP, Spence M, Stierwalt AGJ. Using webcam interactive technology to implement treatment for severe apraxia: a case example. Journal of Medical Speech-Language Pathology. 2010; 18(4):71.

Cherney, LR.; Babbitt, EM.; Kim, K-Y.; Hurwitz, R.; Ngampatipatpong, N.; van Vuuren, S. Aphasia treatment over the internet: a randomized placebo-controlled clinical trial. Clinical Aphasiology Conference; Fort Lauderdale, FL. 2011.

Cherney LR, Kaye RC, Hitch RS. The Best of Both Worlds: Combining Synchronous and Asynchronous Telepractice in the Treatment of Aphasia. Perspectives on Neurophysiology and Neurogenic Speech and Language Disorders. 2011; 21:83-93.

Dechêne L, Tousignant M, Macoir J, Héroux S, Brière S, Pagé C. Simulated in-home teletreatment for anomia. International Journal of Telerehabilitation. 2011; 3(2):3-10. [PubMed: 25945183]

Friedman RB, Lott SN. Rapid word identification in pure alexia is lexical but not semantic. Brain and Language. 2000; 72(3):219-37.10.1006/brln.2000.2286 [PubMed: 10764518]

Friedman RB, Sample DM, Lott SN. The role of level of representation in the use of paired associate learning for rehabilitation of alexia. Neuropsychologia. 2002; 40(2):223-34. [PubMed: 11640944] 
Furnas DW, Edmonds La. The effect of computerised Verb Network Strengthening Treatment on lexical retrieval in aphasia. Aphasiology. 2014; 00(00):1-20.10.1080/02687038.2013.869304

Georgeadis A, Brennan DM, Barker L, Baron CR. Telerehabilitation and its effect on story retelling by adults with neurogenic communication disorders. Aphasiology. 2004; 18(5-7):639652.10.1080/02687030444000075

Goldberg S, Haley KL, Jacks A. Script training and generalization for people with aphasia. American Journal of Speech-Language Pathology. 2012; 21(August):222238.10.1044/1058-0360(2012/11-0056)a [PubMed: 22442283]

Hill A, Theodoros DG, Russell TG, Ward EC. Using telerehabilitation to assess apraxia of speech in adults. International Journal of Language \& Communication Disorders/Royal College of Speech \& Language Therapists. 2009; 44(5):731-47.10.1080/13682820802350537 [PubMed: 18821157]

Howard D, Patterson K. The Pyramids and Palm Trees Test: A test of semantic access from words and pictures. 1992

Kully D. Telehealth in speech pathology: applications to the treatment of stuttering. Journal of Telemedicine and Telecare. 2000; 6(Supplement 2):s2:39-s2:41. [PubMed: 10975081]

Kurland J, Cortes CR, Sperling AJ, Lott SN, Lacey EH, Orchinik L, Friedman RB. Functional reorganization supporting learning and maintenance in a case of phonologic alexia. Brain and Language. 2007; 103(1-2):99-100.10.1016/j.bandl.2007.07.063

Kurland J, Cortes CR, Wilke M, Sperling AJ, Lott SN, Tagamets Ma, Friedman RB. Neural Mechanisms Underlying Learning following Semantic Mediation Treatment in a case of Phonologic Alexia. Brain Imaging and Behavior. 2008; 2(3):147-162.10.1007/s11682-008-9027-2 [PubMed: 20119495]

Lott SN, Carney AS, Glezer LS, Friedman RB. Overt use of a tactile-kinesthetic strategy shifts to covert processing in rehabilitation of letter-by-letter reading. Aphasiology. 2010; 24(11):14241442.10.1080/02687030903580333 [PubMed: 21170161]

Lott SN, Sample DM, Oliver RT, Lacey EH, Friedman RB. A patient with phonologic alexia can learn to read "much" from "mud pies". Neuropsychologia. 2008; 46(10):2515-23.10.1016/ j.neuropsychologia.2008.04.004 [PubMed: 18513760]

Mashima PA, Doarn CR. Overview of telehealth activities in speech-language pathology. Telemedicine and E-Health. 2008; 14(10):1101-17.10.1089/tmj.2008.0080 [PubMed: 19119834]

Mortley J, Wade J, Enderby P. Superhighway to promoting a client-therapist partnership? Using the Internet to deliver word-retrieval computer therapy, monitored remotely with minimal speech and language therapy input. Aphasiology. 2004; 18(3):193-211.10.1080/02687030344000553

Mortley J, Wade J, Enderby P, Hughes A. Effectiveness of computerised rehabilitation for long-term aphasia: a case series study. British Journal of General Practice. 2004; 54(508):856-7. [PubMed: 15527613]

Ong YH, Brown MM, Robinson P, Plant GT, Husain M, Leff AP. Read-Right: a "web app" that improves reading speeds in patients with hemianopia. Journal of Neurology. 2012; 259(12):26115.10.1007/s00415-012-6549-8 [PubMed: 22688568]

Theodoros DG, Hill A, Russell TG, Ward EC, Wootton R. Assessing acquired language disorders in adults via the Internet. Telemedicine and E-Health. 2008; 14(6):552-559. [PubMed: 18729754]

Tindall L, Huebner R, Stemple J, Kleinert H. Videophone-delivered voice therapy: A comparative analysis of outcomes to traditional delivery for adults with Parkinson's disease. Telemedicine and E-Health. 2008; 14(10):1070-1077. [PubMed: 19119829]

Van Casteren M, Davis MH. Match: a program to assist in matching the conditions of factorial experiments. Behavior Research Methods. 2007; 39(4):973-8. Retrieved from http:// www.ncbi.nlm.nih.gov/pubmed/18183914. [PubMed: 18183914]

Vaughn GR. Tel-communicology: health-care delivery system for persons with communicative disorders. ASHA. 1976; 18(1):13-7. Retrieved from http://www.ncbi.nlm.nih.gov/pubmed/ 1247464. [PubMed: 1247464] 


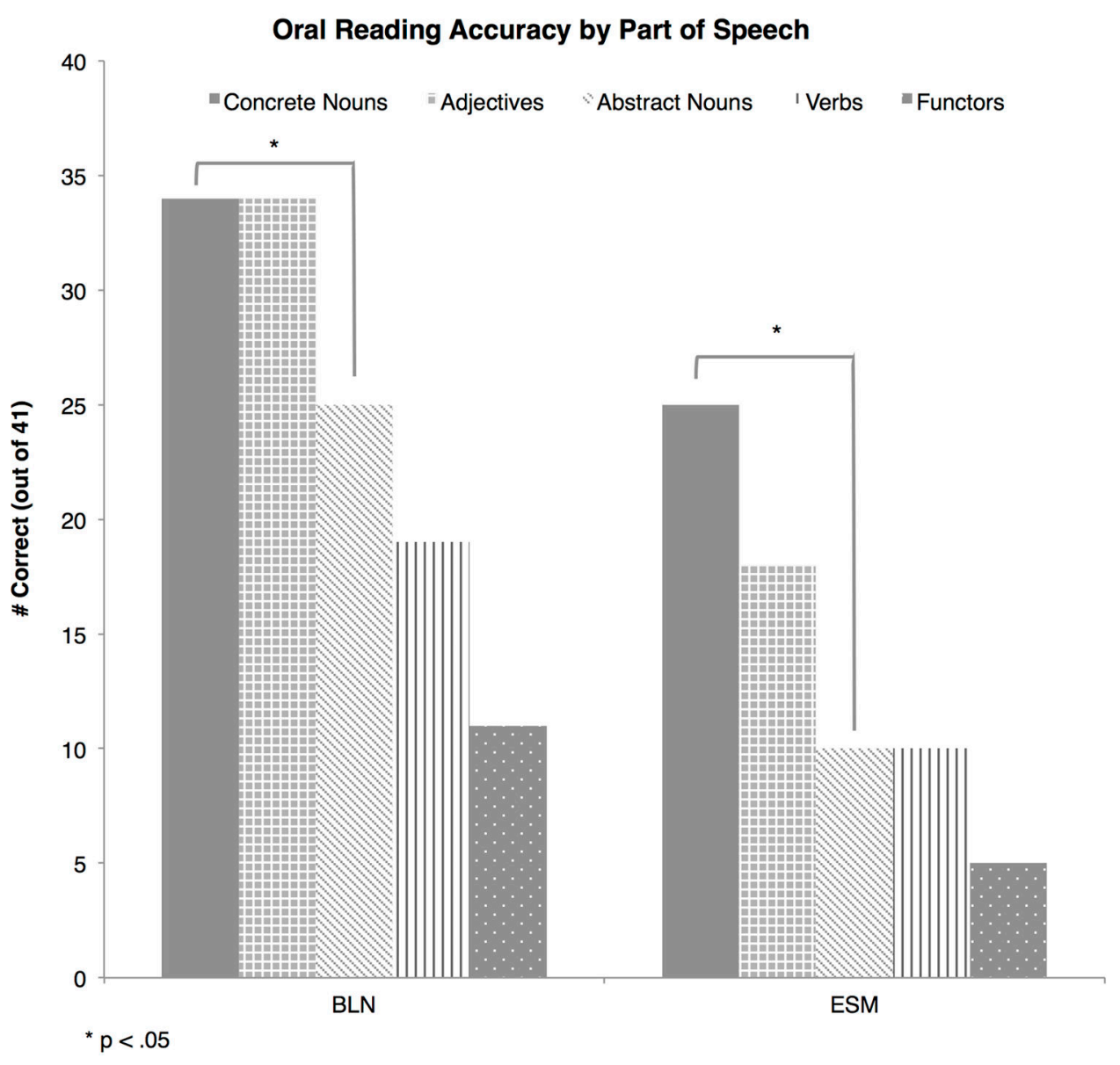

Figure 1.

Participants' oral reading accuracy by part of speech. 


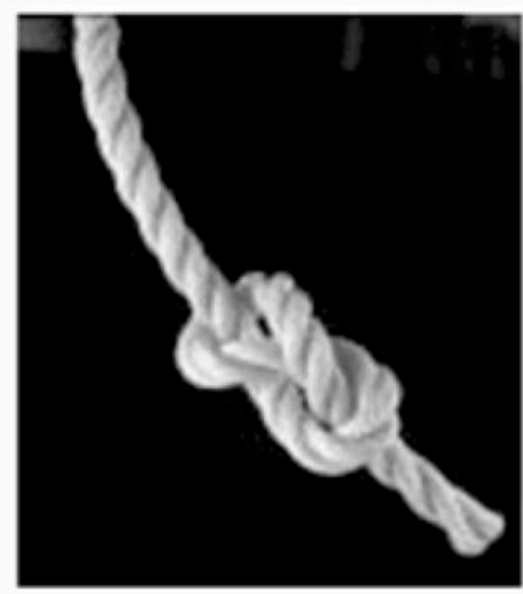

Figure 2.

Screenshot of telerehab software during treatment, after clicking "flip." The target word appears on the left and its homophonic relay word appears on the right, underneath its picture. 


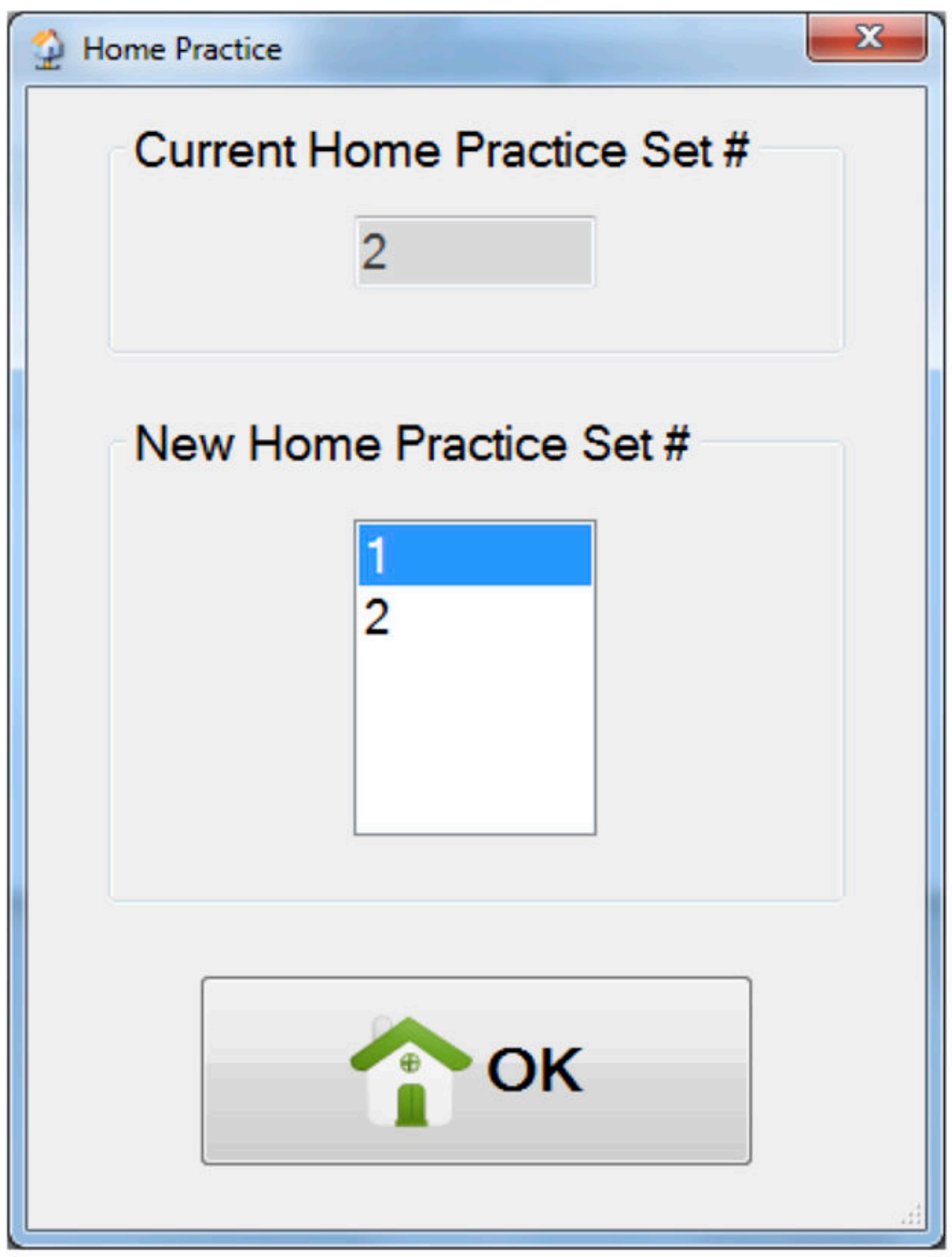

Figure 3.

Home practice selection menu of telerehab software. 

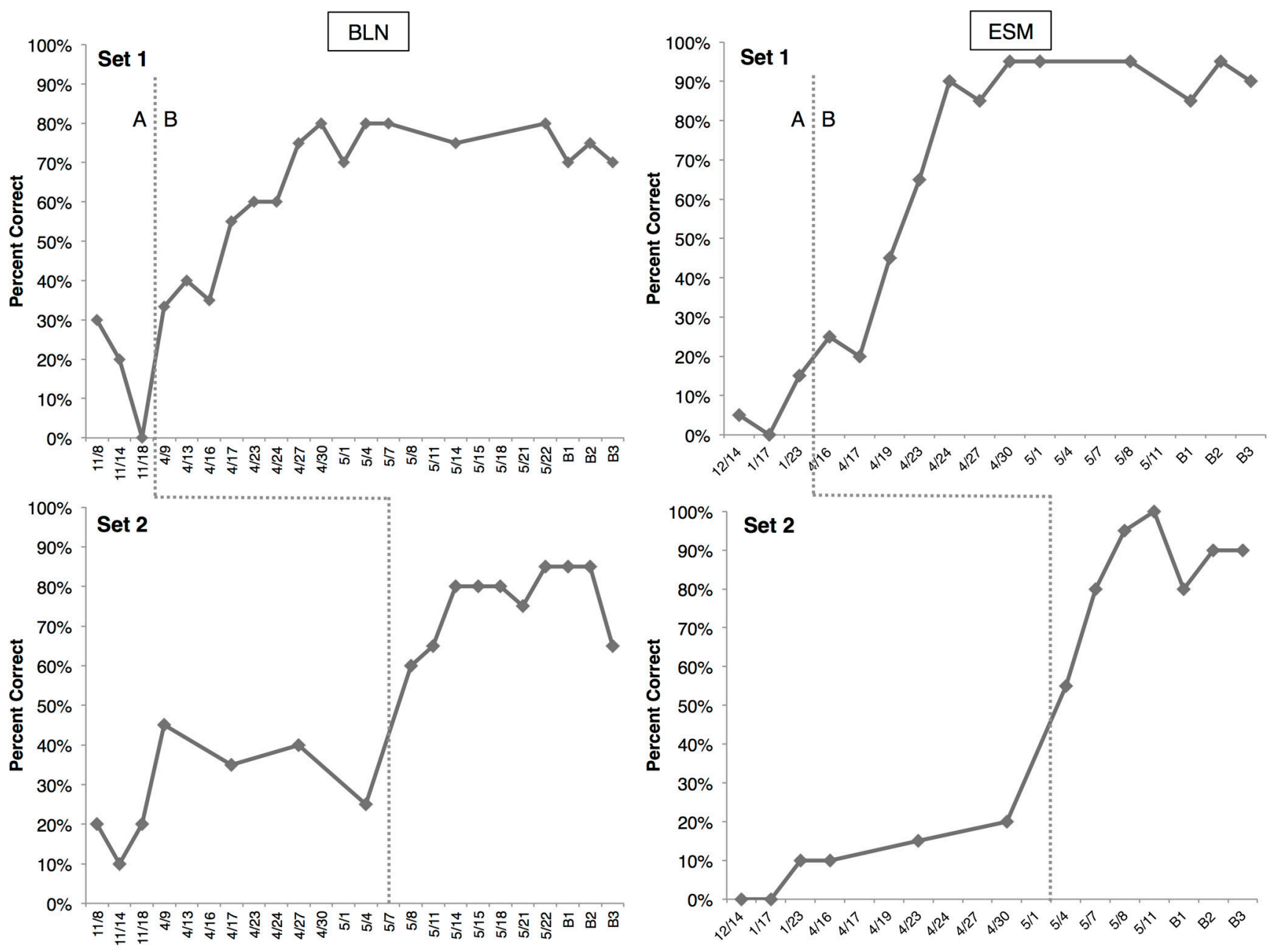

Figure 4.

Results of treatment intervention. Two study phases are represented: A, baseline phase, and $\mathrm{B}$, treatment phase. 


\section{BLN Oral Reading Accuracy}

-Pre-Test $\quad$ Post-Test

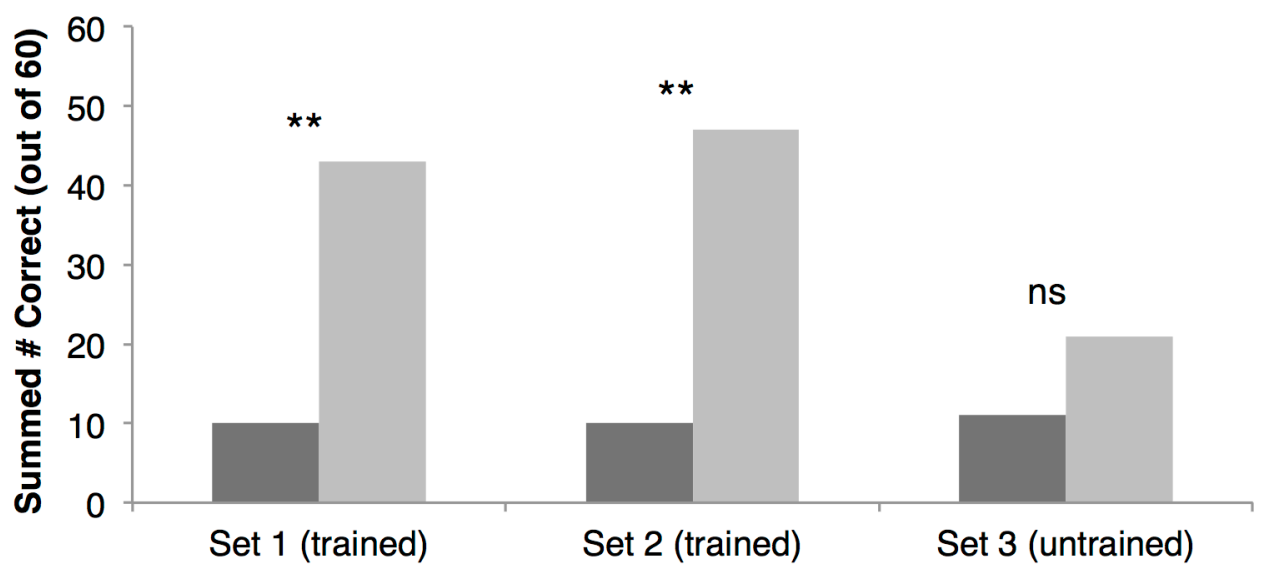

\section{ESM Oral Reading Accuracy}

-Pre-Test $\quad$ Post-Test

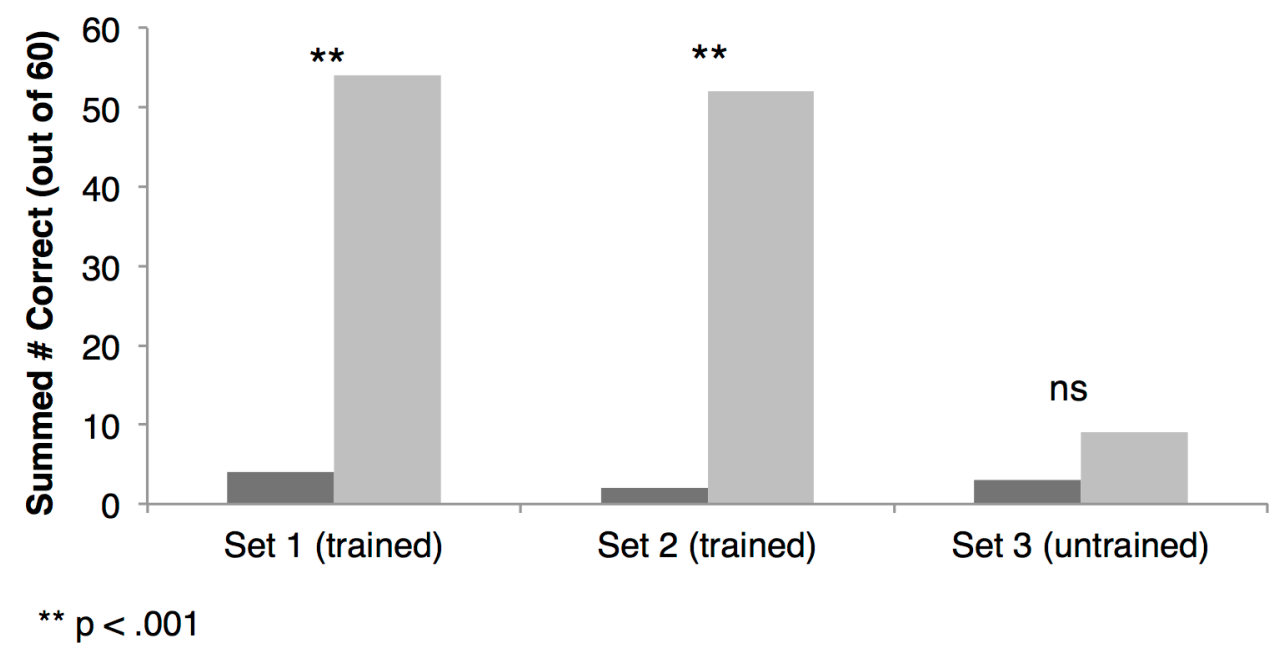

Figure 5.

Performance on target items pre- and post-treatment. 
Time to criterion by treatment setting

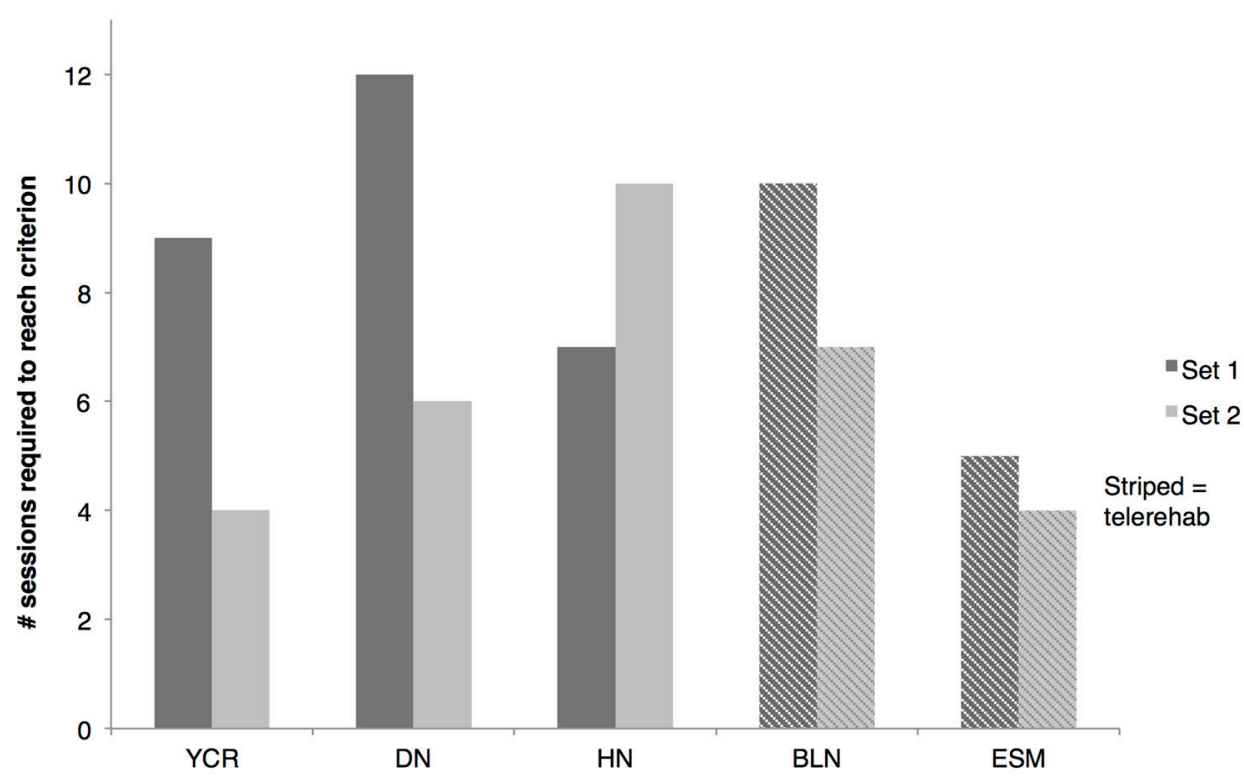

Figure 6.

Comparison of in-person and telerehab SemMed treatment. 


\section{Table 1}

Training set parameters for BLN and ESM.

\begin{tabular}{|c|c|c|c|}
\hline \multicolumn{4}{|l|}{ BLN } \\
\hline & Set $1(n=20)$ & Set $2(n=20)$ & Set $3(n=20)$ \\
\hline Avg Celex_W & 2828 & 2937 & 2385 \\
\hline Length in Syllables & 1.15 & 1.15 & 1.15 \\
\hline Length in Phonemes 1 & 3.05 & 3.15 & 2.85 \\
\hline Length in Letters & 3.65 & 3.85 & 3.45 \\
\hline Accuracy over 3 baselines (60) & 11 & 11 & 11 \\
\hline \# Exact Homophones & 10 & 9 & 10 \\
\hline \multicolumn{4}{|l|}{ ESM } \\
\hline & Set $1(n=20)$ & Set $2(n=20)$ & Set $3(n=20)$ \\
\hline Avg Celex_W & 1154 & 1128 & 1033 \\
\hline Length in Syllables & 1.00 & 1.15 & 1.05 \\
\hline Length in Phonemes & 2.80 & 3.00 & 2.85 \\
\hline Length in Letters & 3.95 & 3.95 & 3.65 \\
\hline Accuracy over 3 baselines (60) & 4 & 2 & 3 \\
\hline \# Exact Homophones & 16 & 15 & $1^{2}$ \\
\hline
\end{tabular}


Table 2

Time and distance required for participants to travel to GUMC

\begin{tabular}{lrr}
\hline & ESM & BLN \\
Round trip & & \\
Miles & 59.2 & 20.2 \\
Time (min) & 80 & 46 \\
Number of sessions & & \\
Pre-test \& setup & 6 & 7 \\
Telerehab & 13 & 19 \\
Post-test & 4 & 4 \\
TOTAL & 23 & 30 \\
Projected travel & & \\
Miles & 1361.6 & 606 \\
Time (min) & 1840 & 1380 \\
Actual travel (pre and post testing) & & \\
Miles & 592.0 & 222.2 \\
Time (min) & 800 & 506 \\
Savings & & \\
Miles & 769.6 & 383.8 \\
Time (min) & 1040 & 874 \\
Time (h) & 17.3 & 14.6 \\
\hline & & \\
\hline
\end{tabular}

